\title{
Asymptotic analysis of Bragg fibers
}

\author{
Yong Xu, Reginald K. Lee, and Amnon Yariv
}

Department of Applied Physics, California Institute of Technology, MS 128-95, Pasadena, California 91125

Received July 31, 2000

\begin{abstract}
Using an asymptotic analysis, we obtain an eigenvalue equation for the general mode dispersion in Bragg fibers. The asymptotic analysis is applied to calculate the dispersion relation and the field distribution of $\mathrm{TE}$ modes in a Bragg fiber. We compare the asymptotic results with exact solutions and find excellent agreement between them. This asymptotic approach greatly simplifies the analysis and design of Bragg fibers. () 2000 Optical Society of America
\end{abstract}

OCIS codes: $060.2280,230.7370,260.2110,060.2310$.

Bragg fibers, in which light confinement is due to cylindrical Bragg reflection instead of total internal reflection, were proposed by Yeh et al. ${ }^{1}$ An especially appealing feature of these fibers is the possibility of guiding light in an air core, which has attracted considerable recent interest. ${ }^{2-5}$ Potential advantages of air-core fibers are lower absorption loss and higher threshold power for nonlinear effects. These fibers also offer other possibilities such as atom guiding by optical waves. ${ }^{3}$

In the original proposal and analysis of Bragg fibers, ${ }^{1}$ it was shown that confined modes exist in a Bragg fiber that consists of a low-index core, including air, surrounded by a suitably designed alternating cladding of high- and low-refractive-index media (see Fig. 1). An experimental demonstration of waveguiding in Bragg fibers was recently reported. ${ }^{4}$ The exact theoretical analysis of Bragg fibers in Ref. 1 is considerably more complicated than that of planar Bragg waveguides. ${ }^{6}$ One main reason is that the Bloch theorem no longer applies to cylindrically symmetric geometries such as Bragg fibers. Therefore it is difficult to find an equation that determines the confined modes. Instead, we must treat the confined modes as quasi modes and find the guided modes by minimizing the radiation loss, which can be quite complicated. ${ }^{1}$ In this Letter we develop a formalism to analyze guided modes in Bragg fibers in the asymptotic limit. We find that the asymptotic approach greatly simplifies the problem and provides an excellent approximation to the exact solution, even when the radius of the low-index core is relatively small. Therefore we expect that the results in this Letter will greatly facilitate the design and analysis of Bragg fibers.

We consider a Bragg fiber composed of a lowindex core (refractive index $n_{c}$ ) surrounded by pairs of high- and low-refractive-index layers with $n_{1}$ and $n_{2}$, respectively. Other parameters of the Bragg fiber are defined in Fig. 1. For guided modes we can factor out the temporal and the $z$ dependence as $\exp [i(\beta z-\omega t)]$ and the azimuthal dependence as $\cos (l \theta)$ or $\sin (l \theta){ }^{1}$ If we use the asymptotic expressions ${ }^{7}$ for the Bessel functions at $k r \rightarrow \infty$ to describe the cladding fields, the radial dependence of $E_{z}$ and $H_{z}$ becomes

$$
\begin{aligned}
& E_{z}=a_{c} J_{l}\left(k_{c} r\right) \quad 0<r<\rho_{1}, \\
& E_{z}=\frac{a_{n} \exp \left[i k_{1}\left(r-\rho_{n}\right)\right]+b_{n} \exp \left[-i k_{1}\left(r-\rho_{n}\right)\right]}{\sqrt{k_{1} r}}, \\
& \rho_{n}<r<\rho_{n}+l_{1}, \\
& E_{z}=\frac{a_{n}{ }^{\prime} \exp \left[i k_{2}\left(r-\rho_{n}{ }^{\prime}\right)\right]+b_{n}{ }^{\prime} \exp \left[-i k_{2}\left(r-\rho_{n}{ }^{\prime}\right)\right]}{\sqrt{k_{2} r}}, \\
& \rho_{n}{ }^{\prime}<r<\rho_{n}{ }^{\prime}+l_{2}, \quad(1 \mathrm{a}) \\
& H_{z}=c_{c} J_{l}\left(k_{c} r\right), \quad \rho_{1}, \\
& H_{z}=\frac{c_{n} \exp \left[i k_{1}\left(r-\rho_{n}\right)\right]+d_{n} \exp \left[-i k_{1}\left(r-\rho_{n}\right)\right]}{\sqrt{k_{1} r}}, \\
& H_{z}=\frac{c_{n}{ }^{\prime} \exp \left[i k_{2}\left(r-\rho_{n}{ }^{\prime}\right)\right]+d_{n}{ }^{\prime} \exp \left[-i k_{2}\left(r-\rho_{n}{ }^{\prime}\right)\right]}{\sqrt{k_{2} r}}, \\
& \rho_{n}{ }^{\prime}<r<\rho_{n}{ }^{\prime}+l_{2}, \quad(1 \mathrm{~b})
\end{aligned}
$$

where $k_{c}=\left[n_{c}{ }^{2}(\omega / c)^{2}-\beta^{2}\right]^{1 / 2}, k_{1}=\left[n_{1}{ }^{2}(\omega / c)^{2}-\right.$ $\left.\beta^{2}\right]^{1 / 2}$, and $k_{2}=\left[n_{2}{ }^{2}(\omega / c)^{2}-\beta^{2}\right]^{1 / 2}$.

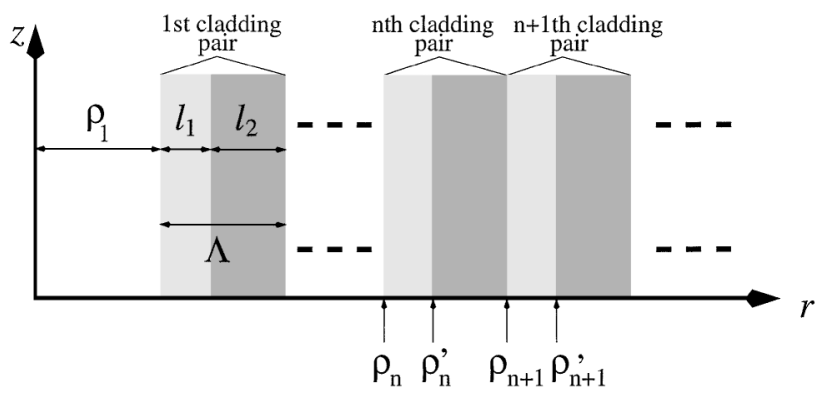

Fig. 1. Schematic of the $r-z$ cross section of a Bragg fiber. The fiber core has refractive index $n_{c}$ and radius $\rho_{1}$. The fiber cladding is composed of pairs of alternating layers of high- and low-index material. The high-index layer has refractive index $n_{1}$ and thickness $l_{1}$. The low-index layer has refractive index $n_{2}$ and thickness $l_{2}$. 
With $E_{z}$ and $H_{z}$ known, other field components can be easily found from the derivatives of $E_{z}$ and $H_{z}{ }^{1}$ The boundary conditions require $E_{z}, E_{\theta}, H_{z}$, and $H_{\theta}$ to be continuous at the interface between two adjacent dielectric layers. Keeping only the leading terms for $E_{\theta}$ and $H_{\theta}$ in the asymptotic limit, we find the following matrix relations:

$$
\begin{aligned}
& \left(\begin{array}{l}
a_{n+1} \\
b_{n+1}
\end{array}\right)=\left[\begin{array}{cc}
A_{\mathrm{TM}} & B_{\mathrm{TM}} \\
B_{\mathrm{TM}} & A_{\mathrm{TM}}
\end{array}\right]\left(\begin{array}{l}
a_{n} \\
b_{n}
\end{array}\right), \\
& \left(\begin{array}{c}
c_{n+1} \\
d_{n+1}
\end{array}\right)=\left[\begin{array}{cc}
A_{\mathrm{TE}} & B_{\mathrm{TE}} \\
B_{\mathrm{TE}} & A_{\mathrm{TE}} *
\end{array}\right]\left(\begin{array}{c}
c_{n} \\
d_{n}
\end{array}\right),
\end{aligned}
$$

where $A_{\mathrm{TE}}, B_{\mathrm{TE}}, A_{\mathrm{TM}}$, and $B_{\mathrm{TM}}$ are defined as

$$
\begin{aligned}
A_{\mathrm{TE}}= & \exp \left(i k_{1} l_{1}\right)\left[i \frac{k_{1}{ }^{2}+k_{2}{ }^{2}}{2 k_{1} k_{2}} \sin \left(k_{2} l_{2}\right)+\cos \left(k_{2} l_{2}\right)\right] \\
B_{\mathrm{TE}}= & i \exp \left(-i k_{1} l_{1}\right) \frac{k_{1}{ }^{2}-k_{2}{ }^{2}}{2 k_{1} k_{2}} \sin \left(k_{2} l_{2}\right) \\
A_{\mathrm{TM}}= & \exp \left(i k_{1} l_{1}\right)\left[i \frac{n_{2}{ }^{4} k_{1}{ }^{2}+n_{1}{ }^{4} k_{2}{ }^{2}}{2 n_{1}{ }^{2} n_{2}{ }^{2} k_{1} k_{2}} \sin \left(k_{2} l_{2}\right)\right. \\
& \left.+\cos \left(k_{2} l_{2}\right)\right], \\
B_{\mathrm{TM}}= & i \exp \left(-i k_{1} l_{1}\right) \frac{n_{2}{ }^{4} k_{1}{ }^{2}-n_{1}{ }^{4} k_{2}{ }^{2}}{2 n_{1}{ }^{2} n_{2}{ }^{2} k_{1} k_{2}} \sin \left(k_{2} l_{2}\right)
\end{aligned}
$$

Since $A_{\mathrm{TE}}, B_{\mathrm{TE}}, A_{\mathrm{TM}}$, and $B_{\mathrm{TM}}$ are the same for all cladding layers, we can apply the Bloch theorem to the cladding fields:

$$
\begin{aligned}
\left(\begin{array}{l}
a_{n+1} \\
b_{n+1}
\end{array}\right) & =\exp \left(i K_{\mathrm{TM}} \Lambda\right)\left(\begin{array}{l}
a_{n} \\
b_{n}
\end{array}\right), \\
\left(\begin{array}{c}
c_{n+1} \\
d_{n+1}
\end{array}\right) & =\exp \left(i K_{\mathrm{TE}} \Lambda\right)\left(\begin{array}{c}
c_{n} \\
d_{n}
\end{array}\right), \\
\exp \left(i K_{\mathrm{TM}} \Lambda\right) & =\operatorname{Re}\left(A_{\mathrm{TM}}\right) \pm\left\{\left[\operatorname{Re}\left(A_{\mathrm{TM}}\right)\right]^{2}-1\right\}^{1 / 2}, \\
\exp \left(i K_{\mathrm{TE}} \Lambda\right) & =\operatorname{Re}\left(A_{\mathrm{TE}}\right) \pm\left\{\left[\operatorname{Re}\left(A_{\mathrm{TE}}\right)\right]^{2}-1\right\}^{1 / 2}
\end{aligned}
$$

These results clearly indicate that in the asymptotic limit the properties of Bragg fiber cladding resemble those of planar Bragg stacks. ${ }^{6}$ Consequently, many results of planar Bragg stacks can be directly applied to Bragg fibers. For example, the condition $\operatorname{Re}\left(A_{\mathrm{TE}}\right)>1$ indicates the TE Bragg reflection gap, in which $\exp \left(i K_{\mathrm{TE}} \Lambda\right)$ becomes a real number. Also, to achieve optimal confinement in Bragg fibers, we should choose $l_{1}$ and $l_{2}$ such that $k_{1} l_{1}=k_{2} l_{2}=\pi / 2$ (i.e., quarter-wave layers).

To obtain the solution for propagation constant $\beta$ we need to match the values of $E_{z}, E_{\theta}, H_{z}$, and $H_{\theta}$ at $r=\rho_{1}$, which gives us

$$
\begin{gathered}
\frac{\omega^{2}}{c^{2}} n_{c}{ }^{2}\left[\frac{J_{l}^{\prime}\left(k_{c} \rho_{1}\right)}{J_{l}\left(k_{c} \rho_{1}\right)}+i \frac{k_{c} n_{1}^{2} \exp \left(i K_{\mathrm{TM}} \Lambda\right)-A_{\mathrm{TM}}-B_{\mathrm{TM}}}{k_{1} n_{c}{ }^{2} \exp \left(i K_{\mathrm{TM}} \Lambda\right)-A_{\mathrm{TM}}+B_{\mathrm{TM}}}\right] \\
\times\left[\begin{array}{cc}
\frac{J_{l}^{\prime}\left(k_{c} \rho_{1}\right)}{J_{l}\left(k_{c} \rho_{1}\right)}+i \frac{k_{c}}{k_{1}} & \frac{\exp \left(i K_{\mathrm{TE}} \Lambda\right)-A_{\mathrm{TE}}-B_{\mathrm{TE}}}{\exp \left(i K_{\mathrm{TE}} \Lambda\right)-A_{\mathrm{TE}}+B_{\mathrm{TE}}}
\end{array}\right] \\
=\frac{\beta^{2} l^{2}}{k_{c}^{2} \rho_{1}{ }^{2}}
\end{gathered}
$$

It becomes clear that for any $l \neq 0$ the guided modes are a mixture of TE and TM modes. Only for the special case $l=0$ can the guided modes be classified as either TE or TM modes.

Equation (5) governs the general mode dispersion in any Bragg fiber. A natural question arises: How accurate is this asymptotic result? It may seem that asymptotic approximations are only valid for a core radius of at least several wavelengths. However, such is not the case, as will be shown by the following example: To simplify our analysis we restrict ourselves to the TE modes. The low-index core of the fiber is air $\left(n_{c}=1\right)$, and the high and low refractive indices of the fiber cladding are $n_{1}=3$ and $n_{2}=1.5$, respectively. We choose the radius of hollow fiber core to be $\rho_{1}=1 \mu \mathrm{m}$, and the fiber cladding parameters are $l_{1}=0.130 \mu \mathrm{m}$ and $l_{2}=0.265 \mu \mathrm{m}$. These parameters are chosen so that the Bragg fiber cladding forms a quarter-wave stack for $\lambda=1.55 \mu \mathrm{m}$ light. For the TE mode with $l=0$, Eq. (5) is simplified into

$$
\frac{J_{0}^{\prime}\left(k_{c} \rho_{1}\right)}{J_{0}\left(k_{c} \rho_{1}\right)}+i \frac{k_{c}}{k_{1}} \frac{\exp \left(i K_{\mathrm{TE}} \Lambda\right)-A_{\mathrm{TE}}-B_{\mathrm{TE}}}{\exp \left(i K_{\mathrm{TE}} \Lambda\right)-A_{\mathrm{TE}}+B_{\mathrm{TE}}}=0 .
$$

Within the spectral range of $1.4 \mu \mathrm{m}<\lambda<1.6 \mu \mathrm{m}$, the Bragg fiber supports a single TE mode, whose propagation constant $\beta$ is calculated with Eq. (6) and plotted in Fig. 2 together with the exact results obtained with the method reported in Ref. 1 . The agreement between the asymptotic results and exact solutions is excellent, with the difference between the

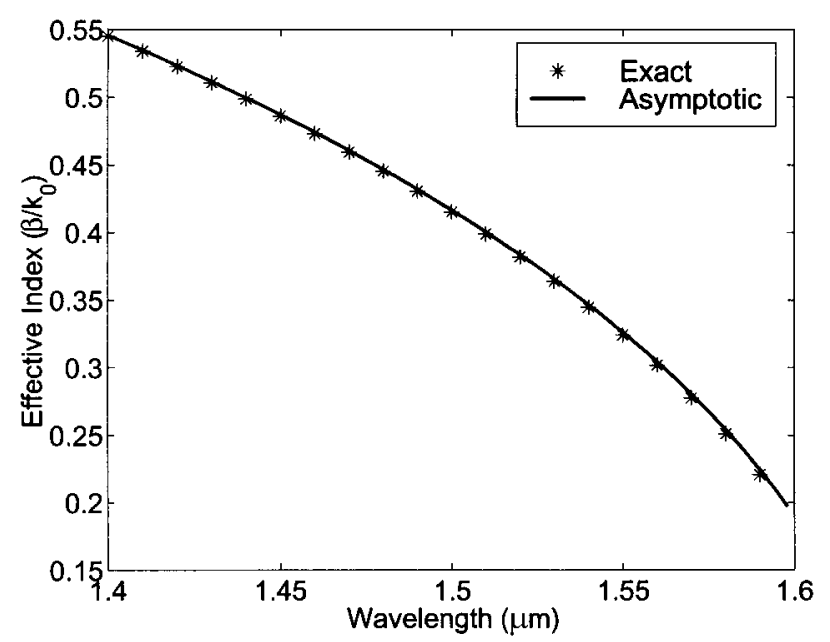

Fig. 2. Dispersion of the fundamental TE mode in an air-core Bragg fiber. The parameters of the Bragg fiber are $n_{c}=1, \rho_{1}=1 \mu \mathrm{m}, n_{1}=3.0, l_{1}=0.130 \mu \mathrm{m}, n_{2}=1.5$, and $l_{2}=0.265 \mu \mathrm{m}$. 

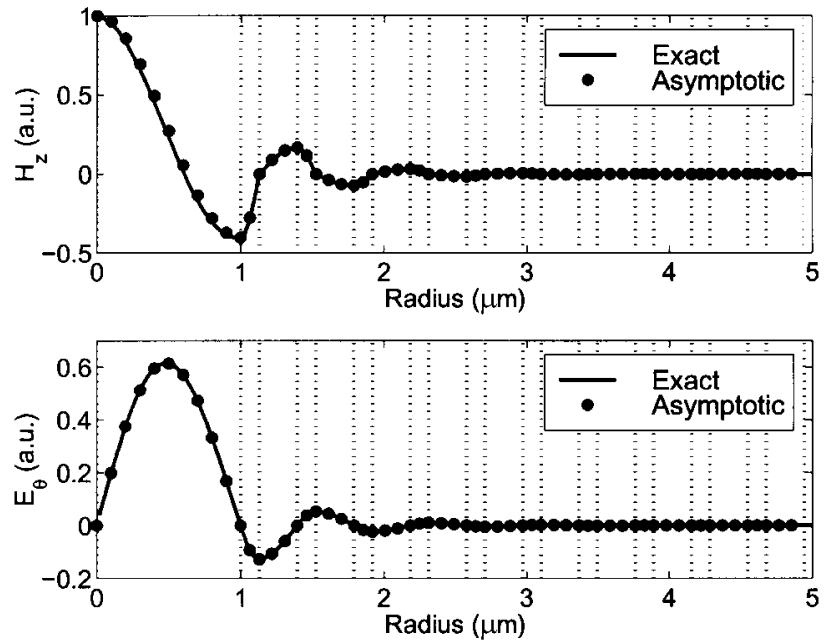

Fig. 3. $H_{z}$ and $E_{\theta}$ field of the guided TE mode at $\lambda=$ $1.55 \mu \mathrm{m}$ in the same Bragg fiber as before. The dotted lines indicate cladding interfaces.

two being less than $2 \%$. Considering the small air-core radius, it is somewhat surprising that the asymptotic approximation performs so well. In our example, $k_{c} \rho_{1}$ is approximately 3.8. Owing to the high refractive index of the first layer, $k_{1} \rho_{1}$ is much larger, typically $\sim 12$. In the exact analysis, the fields in the first cladding layer are described by $J_{0}\left(k_{1} r\right)$ and $Y_{0}\left(k_{1} r\right) .{ }^{1} \quad$ At $x \approx 12$, the asymptotic approximations for $J_{0}(x)$ and $Y_{0}(x)$ are already quite accurate. This explains why the asymptotic limit provides such a good approximation even for relatively small fiber cores. We point out that cladding layers with a large refractive index have been experimentally fabricated, where cladding indices $n_{1}=4.6$ and $n_{2}=1.59$ are used. ${ }^{4}$

Notice that in Fig. 2 the effective index $\beta / k_{0}$ is always less than unity, which is a unique feature of guiding light in hollow-core Bragg fibers. The dispersion of the TE mode, as can be seen from Fig. 2, is quite strong, which is a direct results of the small air core. A larger air core reduces the dispersion of the Bragg fiber. At the same time, however, the Bragg fiber may support higher-order modes. Therefore, in the design of Bragg fibers, we need to carefully balance the requirements of single-mode operation and small dispersion. The asymptotic results in this Letter provide an accurate and fast way of analyzing this problem.

It is also of interest to calculate the field distribution of the guided modes. For a confined TE mode, $E_{z}=0$ and $\exp \left(i K_{\mathrm{TE}} \Lambda\right)$, as given by Eq. (4), should be a real number with an absolute value less than 1 . With these observations, from Eqs. (2) and (4) we find

$$
\left(\begin{array}{c}
c_{n} \\
d_{n}
\end{array}\right)=C \exp \left[i(n-1) K_{\mathrm{TE}} \Lambda\right]\left[\begin{array}{c}
B_{\mathrm{TE}} \\
\exp \left(i K_{\mathrm{TE}} \Lambda\right)-A_{\mathrm{TE}}
\end{array}\right]
$$

$$
\begin{aligned}
& \left(\begin{array}{c}
c_{n}{ }^{\prime} \\
d_{n^{\prime}}{ }^{\prime}
\end{array}\right)= \\
& \frac{1}{2} \sqrt{\frac{k_{2}}{k_{1}}}\left[\begin{array}{c}
\frac{k_{1}+k_{2}}{k_{1}} \exp \left(i k_{1} l_{1}\right) \frac{k_{1}-k_{2}}{k_{1}} \exp \left(-i k_{1} l_{1}\right) \\
\frac{k_{1}-k_{2}}{k_{1}} \exp \left(i k_{1} l_{1}\right) \frac{k_{1}+k_{2}}{k_{1}} \exp \left(-i k_{1} l_{1}\right)
\end{array}\right] \\
& \times\left(\begin{array}{c}
c_{n} \\
d_{n}
\end{array}\right),
\end{aligned}
$$

where the overall constant $C$ is determined by the condition of $c_{c}=1$ in Eq. (1), which gives

$$
C=\frac{J_{0}\left(k_{c} \rho_{1}\right) \sqrt{k_{1} \rho_{1}}}{\exp \left(i K_{\mathrm{TE}} \Lambda\right)-A_{\mathrm{TE}}+B_{\mathrm{TE}}} .
$$

The $E_{\theta}$ component can be easily found from the derivative of $H_{z} \cdot{ }^{1}$ We calculate both $H_{z}$ and the $E_{\theta}$ field for the guided TE mode at $\lambda=1.55$ in the Bragg fiber studied in Fig. 2. In Fig. 3 we show the asymptotic solutions, together with the exact results obtained with the method presented in Ref. 1. The two results agree well with each other, which again confirms the accuracy of the asymptotic analysis. A particularly interesting feature of Fig. 3 is that the field decays to almost zero within only a few pairs of cladding layers, a direct result of large index contrast between the cladding materials. In fact, a short calculation of $\exp \left(i K_{\mathrm{TE}} \Lambda\right)$ shows that it is possible to achieve $0.2 \mathrm{~dB} / \mathrm{km}$ loss with less than 20 pairs of cladding layers.

In conclusion, we have developed an asymptotic formalism that greatly simplifies the analysis of Bragg fibers. Even for an example with relatively small central air core, the asymptotic results agrees well with the exact solutions. If more-accurate results are desired, we can treat the first several cladding layers exactly and use this asymptotic formalism for the rest of the cladding structure.

This research was sponsored by the U.S. Office of Naval Research, whose support is gratefully acknowledged. Y.Xu's e-mail address is yong@its.caltech.edu.

\section{References}

1. P. Yeh, A. Yariv, and E. Marom, J. Opt. Soc. Am. 68, 1196 (1978).

2. M. Miyagi, A. Hongo, Y. Aizawa, and S. Kawakami, Appl. Phys. Lett. 43, 431 (1983).

3. H. Ito, T. Nakata, K. Sakaki, M. Ohtsu, K. I. Lee, and W. Jhe, Phys. Rev. Lett. 76, 4500 (1995).

4. Y. Fink, D. J. Ripin, S. Fan, C. Chen, J. D. Joannopoulos, and E. L. Thomas, J. Lightwave Technol. 17, 2039 (1999).

5. R. F. Cregan, B. J. Mangan, J. C. Knight, T. A. Birks, P. St. J. Russell, P. J. Roberts, and D. C. Allan, Science 285, 1537 (1999).

6. P. Yeh and A. Yariv, Opt. Commun. 19, 427 (1976).

7. J. Mathews and R. L. Walker, Mathematical Methods of Physics (Addison-Wesley, Reading, Mass., 1970). 